\section{ADHS: Aktuelle Empfehlungen aus den USA}

\section{Eine Gruppe von Spezialisten für ADHS der American Academy of Pediatrics hat kürzlich die Aktualisierung der Clinical Practice Guidelines (CPG) für ADHS veröffentlicht.}

N eu und bisher einzigartig im Vergleich $\mathrm{zu}$ anderen Leitlinien über ADHS wurde das Alter der betroffenen Kinder von sechs bis zwölf Jahren auf vier bis 18 Jahre erweitert - und damit dem praktischen Alltag Rechnung getragen. Sechs Schlüsselaussagen konnten nach intensiver Evaluierung von der Spezialistengruppe erstellt werden:

1. Der behandelnde Arzt sollte bei jedem

Kind zwischen vier und 18 Jahren eine ADHS-Diagnostik veranlassen, wenn das Kind Lern- oder Verhaltensprobleme zeigt, zusätzlich zu Symptomen wie Unaufmerksamkeit, Hyperaktivität oder Impulsivität. Diese Aussage bekam Rating B mit starker Empfehlung*

2. Für die Diagnose ADHS soll der Arzt die DSM-IV-Kriterien zugrunde legen. Die Informationen sollen durch Befragung der Eltern, der Erzieher oder Lehrer erfasst werden. Andere Ursachen sollen ausgeschlossen werden (Rating B mit starker Empfehlung).

3. Im Rahmen der Diagnostik sollen Komorbiditäten berücksichtigt werden wie emotionale und Verhaltensstörungen, Lernstörungen und andere neurologische Entwicklungsstörungen sowie Störungen wie Tics und Schlafapnoen (Rating B mit starker Empfehlung).

4. Der Arzt soll ADHS als chronische Erkrankung einstufen und die Betroffenen entsprechend umfassend betreuen unter Einbezug von staatlichen Unterstützungsangeboten (Rating B mit starker Empfehlung).

${ }^{\star}$ Der Leitlinie liegen 8.276 Referenzen zugrunde, die hinsichtlich ihres Evidenzgrades bewertet wurden. Wie international üblich, wurde die Qualität der Aussagen mit A bewertet, wenn sie aufgrund von den wissenschaftlichen Standards entsprechenden Studien (randomisierte placebokontrollierte Studien mit ausreichender Fallzahl) entstanden war. Rating B erhielten randomisierte Studien mit niedrigerem Standard. Beobachtungsstudien mit Kohorten-Design erhielten Rating C, Expertenmeinungen das Rating D.
5. Folgende Therapieempfehlungen werden differenziert nach Alter des betroffenen Kindes aufgelistet:

Bei Kindern von vier bis sechs Jahren ist die Therapie der Wahl ein evaluiertes Elterntraining und eine Anleitung der Erzieher (Rating A mit starker Empfehlung). Bei ausbleibender Wirkung ist eine medikamentöse Therapie mit Methylphenidat (MPH) zu starten. Wenn die psychoedukativen Maßnahmen nicht zur Verfügung stehen, kann eine initiale medikamentöse Therapie mit MPH stattfinden (Rating B mit Empfehlung).

Für Kinder von sechs bis elf Jahren ist die Therapie der ersten Wahl eine Stimulanzientherapie (MPH oder Amphetaminsulfatderivate [AS]) (Rating A mit starker Empfehlung). Psychoedukative Maßnahmen (Eltern- und Lehrertraining) sollten zusätzlich oder auch anstelle stattfinden (Rating B mit Empfehlung).

Für Jugendliche von zwölf bis 18 Jahren stellt eine Stimulanzientherapie die Therapie der ersten Wahl dar (Rating A). Zusätzlich könnte eine Verhaltenstherapie verschrieben werden (Rating C mit Empfehlung).

6. Der Arzt soll die medikamentöse Dosis so titrieren, dass ein maximaler Benefit bei minimaler Nebenwirkung erzielt wird (Rating B mit starker Empfehlung).

Subcommittee on Attention-Deficit/Hyperactivity Disorder; Steering Committee on Quality Improvement and Management, Wolraich M et al. ADHS: clinical practice guideline for the diagnosis, evaluation and treatment of attention-deficit/hyperactivity disorder in children and adolescents. Pediatrics 2011; 128: 1007-22

Kommentar: Erstmals wurden auch Kinder unter sechs Jahren mit in die Therapieempfehlungen einbezogen. Dies macht Sinn, da eine ADHS deutlich vor dem sechsten Geburtstag störungsrelevant sein kann. Die Autoren kommen zu dem Schluss, dass die möglichen Schäden durch eine gestörte Entwicklung höher zu werten seien als mögliche Gefahren durch eine schon zu diesem Zeitpunkt begonnenen Therapie. Die Wirksamkeit der MPH-Therapie ist auch in diesem Alter studienbelegt. Die Autoren weisen aber darauf hin, dass die Medikation nicht für dieses Alter zugelassen ist, die Therapie also Off-Label erfolgt mit den bekannten Risiken für den behandelnden Arzt.

Dies entspricht unserem klinischen Alltag: Jeder Arzt, der viele Kinder mit ADHS betreut, wird eine meist überschaubare Anzahl von Kindern mit ADHS unter sechs Jahren behandeln, bei denen die Störungsrelevanz bereits stark ausgeprägt ist. Diese Kinder erhalten leider erst mithilfe einer Off-Label-Medikation die Chance einer altersadäquaten sozialen Integration. Dabei erleben sie dramatische Entwicklungsfortschritte im sensomotorischen und kognitiven Bereich, da sie unter Therapie erstmals in der Lage sind, sich ausdauernd mit einem Gegenstand zu beschäftigen und komplexe Rollenspiele auszuführen. Auch eine positive Eltern-Kind- sowie Geschwister-Beziehung kann sich wieder entwickeln. Die Leitlinien sollen keinen Freibrief darstellen, jedes Kind mit ADHS unter sechs Jahren medikamentös zu behandeln. Sie sollen aber dazu ermutigen, diesen Schritt zu wagen, wenn die Entwicklung des Kindes gefährdet ist.

Bemerkenswert erscheint es mir auch, dass in den Therapieempfehlungen nicht die von uns so ausufernd eingesetzte Ergotherapie auftaucht. Die Autoren weisen explizit darauf hin, dass die Evidenzlage von verhaltenstherapeutisch orientierte Therapien schwach ist. Lediglich das Elterntraining, das von jedem, der es kann - also auch von einem Ergotherapeuten - ausgeführt wird, hat evidenzbasierte Empfehlungen erhalten.

Zusammenfassend ist die Lektüre der Leitlinien sehr zu empfehlen. Zu hoffen, dass sie unsere verkrusteten Strukturen lockert, ist wahrscheinlich naiv. Ich beneide die Amerikaner darum, dass ADHS in ihrem Land eine Angelegenheit auch der pädiatrischen Gesellschaft ist - insbesondere der akademischen pädiatrischen Gesellschaft und nicht wie in Deutschland aus dem Fortbildungsprogramm der akademischen Pädiatrie weitgehend ausgeschlossen ist.

Dr. Kirsten Stollhoff 